\title{
Calf Circumference Is a Useful Index for Assessing Dysphagia among Community Dwelling Elderly Recipients of Long-Term Care
}

\author{
Yukiko Kurosawa, ${ }_{1}^{1}$ Koji Hara, ${ }^{1}$ Haruka Tohara, ${ }^{1}$ Chizuru Namiki, ${ }^{1}$ \\ Ariya Chantaramanee, ${ }_{1}$ Ayako Nakane, ${ }^{1}$ Kazuharu Nakagawa, ${ }^{1}$ Kohei Yamaguchi, ${ }^{1}$ \\ Kanako Yoshimi, ${ }^{1}$ Junichi Furuya ${ }^{2}$ and Shunsuke Minakuchi ${ }^{1}$ \\ ${ }^{1}$ Department of Gerodontology, Division of Gerontology and Gerodontology, Tokyo Medical and Dental \\ University, Tokyo, Japan \\ ${ }^{2}$ Department of Oral Health Care Sciences for Community and Welfare, Graduate School of Medical and Dental \\ Sciences, Tokyo Medical and Dental University, Tokyo, Japan
}

\begin{abstract}
Dysphagia is a common problem among older adults, causing aspiration pneumonia and malnutrition. It has been reported that calf circumference (CC), an index of nutritional status and physical activity, correlated with dysphagia in acute care hospitals, suggesting that CC can be a useful index for assessing dysphagia. We therefore aimed to explore the association between dysphagia and CC among community elderly people who require long-term care and determined the optimal CC cut-off value for patients with dysphagia. Our cross-sectional study, conducted at Tokyo Metropolis, included 154 participants (65 men) aged $>65$ years (mean age: $80.1 \pm 7.1$ ) who required long-term care and were examined for dental disease and dysphagia during home visiting treatment. Age, body mass index (BMI), mini-nutritional assessment short-form (MNA-SF) score, Barthel index (BI), CC, functional oral intake scale (FOIS), and dysphagia severity scale (DSS) were evaluated. A DSS score $<5$ was defined as dysphagia. To determine the association between $\mathrm{CC}$ and dysphagia, we performed logistic regression analysis and calculated the CC cut-off value for dysphagia. Thirty-seven participants (24.0\%) were diagnosed with dysphagia. The logistic regression analysis showed that the presence of dysphagia was independently associated with $\mathrm{CC}$ after adjusting for age and sex. The CC cut-off value for the presence of dysphagia was $31.0 \mathrm{~cm}$ in men (sensitivity, 0.818; specificity, 0.868) and $29.3 \mathrm{~cm}$ in women (sensitivity, 0.760 ; specificity, 0.859 ). CC is a useful index for assessing dysphagia among community dwelling individuals who require long-term care.
\end{abstract}

Keywords: calf circumference; dysphagia; elderly; malnutrition; mini nutritional assessment short-form Tohoku J. Exp. Med., 2019 July, 248 (3), 201-208. C 2019 Tohoku University Medical Press

\section{Introduction}

As the aging population increases in developed countries such as Japan, optimal health should be promoted to prevent elderly people from requiring nursing care (United Nations 2015). Notably, dysphagia is a common problem in older adults, including hospital and community dwelling elderly people and nursing home residents, as it can cause aspiration pneumonia, malnutrition, and dehydration (Kayser-Jones and Pengilly 1999; Kawashima et al. 2004). In order to prevent nursing home residents from requiring hospitalization due to dysphagia which can negatively affect their general conditions, it is essential to screen elderly people for dysphagia.

Sarcopenia is characterized by the loss of skeletal muscle, strength, and function as part of the aging process and has a high prevalence among nursing home residents (Bauer et al. 2008; Cruz-Jentoft et al. 2010). Sarcopenia affects limb and swallowing muscles, such as the tongue, pharyngeal muscles and suprahyoid muscles, which can lead to a decline in the strength of swallowing muscles (Tamura et al. 2012; Feng et al. 2013; Molfenter et al. 2015; Machida et al. 2017). Sarcopenia is closely related to dysphagia, as $95.5 \%$ of patients with dysphagia have been reported to have sarcopenia (Maeda and Akagi 2016). In addition, a previous study demonstrated that decreased skeletal muscle index was a significant risk factor for reduced swallowing function (Murakami et al. 2015). Given these findings, it is likely that decreased muscle mass has the potential to predict dysphagia.

Anthropometric measurements, which enable us to easily evaluate body composition in clinical settings, are

Received April 17, 2019; revised and accepted July 3, 2019. Published online July 30, 2019; doi: 10.1620/tjem.248.201.

Correspondence: Koji Hara, Department of Gerodontology, Division of Gerontology and Gerodontology, Tokyo Medical and Dental

University, 1-5-45 Yushima, Bunkyo-ku, Tokyo 113-8510, Japan.

e-mail koji19831031@gmail.com 
suitable tools for assessing health of home care patients and residents of nursing homes, and do not require specific and expensive medical equipment. Among anthropometric measurements, calf circumference (CC) is commonly used, because decreased $\mathrm{CC}$ is correlated with loss of muscle mass and malnutrition (Perissinotto et al. 2002; PorteroMcLellan et al. 2010; Kawakami et al. 2015; Matsuo and Yoshimura 2018). Considering that dysphagia is associated with decreased skeletal muscle mass and malnutrition, we hypothesized that $\mathrm{CC}$ has the potential to be a useful screening parameter for dysphagia (Hudson et al. 2000; Tamura et al. 2012; Maeda and Akagi 2016). However, no study to date has investigated the relationship between dysphagia and $\mathrm{CC}$ for individuals who require long-term care. In the present study, we verified the usefulness of CC measurements for predicting dysphagia in community dwelling elderly people and nursing residents who are in long-term care services.

\section{Materials and Methods}

\section{Participants}

We recruited individuals examined by dentists during home visiting treatment session from the gerodontology department of Tokyo Medical Dental University. Data from checkup appointments were extracted regarding the following: reports on gingivitis, dental or denture problems, and dysphagia between July 2016 and November 2018. The dentists were well trained in dysphagia rehabilitation. Eligibility criteria included age $>65$ years, requirement of long-term care, and consent for study participation. In Japan, the social insurance system, established under the Long-term Care Insurance, provides long-term care for elderly patients who require medical care or support for daily activities. The study participants included community-dwelling elderly people in Tokyo Metropolis. We enrolled 158 individuals in this study; four participants who had neuro-muscular diseases and diseases causing bulbar palsy were excluded. The remaining 154 individuals (men: $\mathrm{n}=65$ ) were included in the analysis. Informed consent was obtained from all participants. The study was approved by the ethics committee of the School of Tokyo Medical Dental University (D2014-047).

\section{Procedures}

Assessment: Dentists who perform daily dysphagia rehabilitation in the same department of the Tokyo Medical Dental University, Dental Hospital collected the background data. Medical history, records, dwelling place, and body mass index (BMI) of the participant were collected. Nutritional status was categorized in participants as normal, at risk of malnutrition, and diagnosed with malnutrition based on a mini-nutritional assessment-short form (MNA-SF) (Vellas et al. 2006; Rubenstein et al. 2001). It has been previously reported that MNA-SF has a sensitivity of $98 \%$, specificity of $100 \%$, and diagnostic accuracy of $99 \%$ for predicting undernutrition (Rubenstein et al. 2001). Furthermore, the reliability of the tool has already been verified (Bleda et al. 2002; Baath et al. 2008). ADLs were evaluated using the Barthel index (BI), which consists of an ordinal assessment of ADLs, where lower scores indicate lower physical function (Mahoney and Barthel 1965). In addition, the reliability has been verified (Sainsbury et al. 2005).

A highly reliable assessment tool referred to as the functional oral intake scale (FOIS), was used to evaluate oral intake (Crary et al. 2005). FOIS is a seven-point ordinal scale, where a score of 1 indicates nothing by mouth; 2 , tube dependent with minimal attempts of food or liquid; 3, tube dependent with consistent oral intake of food or liquid; 4, total oral diet of a single consistency; 5, total oral diet with multiple consistencies, but requiring special preparation or compensations; 6 , total oral diet with multiple consistencies without special preparation, but with specific food limitations; and 7, total oral diet without restriction.

Based on a previous study, $\mathrm{CC}$ was measured in the thickest part of the dominant foot using a non-stretch tape (Matsuo and Yoshimura 2018). Participants were then asked to sit on a chair or wheelchair or lie down on a bed with their knees at a $90^{\circ}$ angle and their soles placed on the ground. An average of 3 repetitions was used in the analyses. The reliability of the $\mathrm{CC}$ measurement has been verified (Carmont et al. 2013; Jamaiyah et al. 2008). Measurements including MNA-SF, BI, FOIS and CC were measured by the same dentist who assessed the background data for confounding variables before evaluating of the severity of dysphagia the same day in the participant home. The severity of dysphagia was assessed using the dysphagia severity scale (DSS) by three well trained dentists who were different from the dentists collecting the measurement items above; the DSS assessment was blinded to the value of CC. DSS is a seven-point ordinal scale, where a score of 1 indicates saliva aspiration (unstable medical condition); 2, food aspiration (food aspiration with no effect of compensatory techniques); 3, water aspiration (aspiration of thin liquid); 4, occasional aspiration (possible aspiration or aspiration during chewing or swallowing); 5, oral problems (significant symptoms of oral preparatory or oral phase without aspiration); 6, minimal problems (some symptoms of aspiration); and 7, within normal limits and no symptoms of dysphagia (Nishimura et al. 2015). Twenty-six participants with FOIS level 5 or lower underwent videoendoscopy (VE) in order to have their DSS score assessed. Participants with FOIS levels greater than 5 were evaluated for DSS without VE. Following this, the examiner assessed DSS based on a previous study (Matsuo and Yoshimura 2018). Specifically, after the dentists examined the present and past history, vital signs, consciousness level, cranial nerve problems, and chest and neck auscultation in the participants, they assessed the DSS using a water and food dysphagia screening test and by observing the patient's eating (Tohara et al. 2003). Among participants with FOIS levels $>5$, we suspected that 9 had DSS levels $<4$, and these participants underwent VE; DSS scores 1-4 indicate dysphagia with aspiration. DSS reliability and validity has already been established (Ikeda et al. 2007; Wakabayashi and Sakuma 2014). The DSS was negatively correlated with the eating assessment tool (EAT-10) $(\mathrm{r}=-0.530)$ (Wakabayashi and Sakuma 2014). The inter-class correlation coefficient of the DSS is 0.784 (Ikeda et al. 2007).

\section{Compliance with ethical standards}

The study was approved by the ethics committee of the School of Tokyo Medical Dental University (D2014-047).

\section{Statistical analyses}

An unpaired t-test was used to analyze parametric data, the Wilcoxon signed rank test was used for nonparametric data, and the Chi-square test was used for nominal data to compare participants with and without dysphagia. In addition, the data was used to perform a comparative analysis to assess the differences between gen- 
ders. Correlations among DSS, FOIS, BI, CC, malnutrition, and age were assessed using a bivariate correlation analysis with Spearman's rank test. Following this, a receiver operating characteristic (ROC) curve analysis was performed for men and women to determine the optimal cut-off points. The ROC curves were developed by plotting sensitivity vs. 1-specificity, and the optimal cut off point was defined as the closest point to the upper left-hand corner of the graph (high, sensitivity and low, 1-specificity). After assessing the presence of dysphagia, the sensitivity, specificity, positive predictive value (PPV), and negative predictive value (NPV) were calculated using the optimal cut-off values in both men and women. In addition, the area under the curve was calculated for both men and women. After calculating the cutoff value of $\mathrm{CC}$ in men and women, the association between the cutoff value of $\mathrm{CC}$ and dysphagia was determined using a logistic regression analysis adjusted for age and sex. To select the appropriate model, statistically significant variables $(\mathrm{p}<0.05)$, potential confounding variables, and variables of clinically reasonable combinations were selected, such that multicollinearity would not affect the corresponding logistic regression analysis. All statistical analyses were performed with EZR (Saitama Medical Center, Jichi Medical University, Saitama, Japan), which is a graphical user interface for R (The R Foundation for Statistical Computing, Vienna, Austria).

\section{Sample size calculation}

We calculated our sample size using G*Power software (G*Power 3.1 software; Kiel University, Kiel, Germany). To determine an appropriate effect size, we referred to a previous study about the difference of $\mathrm{CC}$ at discharge between participants with and without dysphagia (effect size, $d=0.62$ ) (Matsuo et al. 2017) Furthermore, the allocation of participants with and without dysphagia was set as 1.3 , based on a previous study that included participants who required long-term medical care (Wakabayashi and Matsushima 2016). Thus, we determined that our sample size should

Table 1. Univariate analysis for dysphagia.

\begin{tabular}{|c|c|c|c|c|}
\hline & $\begin{array}{c}\text { Total } \\
(\mathrm{n}=154)\end{array}$ & $\begin{array}{l}\text { Dysphagia } \\
(n=37)\end{array}$ & $\begin{array}{c}\text { No dysphagia } \\
\quad(n=117)\end{array}$ & $p$ value \\
\hline Age & $80.1 \pm 7.1$ & $81.7 \pm 7.8$ & $79.6 \pm 6.8$ & 0.113 \\
\hline \multicolumn{5}{|l|}{ Sex } \\
\hline Men & $65(42.2)$ & $12(32.4)$ & $53(45.3)$ & 0.053 \\
\hline Women & $89(57.8)$ & $25(67.6)$ & $64(54.7)$ & \\
\hline Disease requiring long term care & & & & $<0.000^{*}$ \\
\hline \multicolumn{5}{|l|}{ n (\%) } \\
\hline Cerebral vascular disease & $61(39.6 \%)$ & $21(56.8 \%)$ & $40(34.2 \%)$ & \\
\hline Dementia & $16(10.4 \%)$ & $8(21.6 \%)$ & $8(6.8 \%)$ & \\
\hline Parkinson disease & $8(5.2 \%)$ & $1(2.7 \%)$ & $7(6.0 \%)$ & \\
\hline Respiratory disease & $7(4.5 \%)$ & $0(0 \%)$ & $7(6.0 \%)$ & \\
\hline Cardiovascular disease & $4(2.6 \%)$ & $1(2.7 \%)$ & $3(2.6 \%)$ & \\
\hline Bone and joint disease & $28(18.2 \%)$ & $0(0 \%)$ & $28(23.9 \%)$ & \\
\hline Cancer & $4(2.6 \%)$ & $1(2.7 \%)$ & $3(2.6 \%)$ & \\
\hline The Other & $27(17.5 \%)$ & $5(13.5 \%)$ & $22(18.8 \%)$ & \\
\hline \multirow[t]{2}{*}{ Body mass index } & 22.12 & 18.8 & 23.1 & $<0.000^{*}$ \\
\hline & $(19.6-25.3)$ & $(17.1-21.5)$ & $(21.1-25.9)$ & \\
\hline \multirow[t]{2}{*}{ Calf circumference $(\mathrm{cm})$} & 32.8 & 27 & 33.8 & $<0.000^{*}$ \\
\hline & $(29.1-35.1)$ & $(22.8-30.5)$ & $(31.3-36.3)$ & \\
\hline MNA-SF, n (\%) & & & & $<0.000^{*}$ \\
\hline Normal & $23(14.9 \%)$ & $19(51.4 \%)$ & $4(3.4 \%)$ & \\
\hline at risk & $53(34.4 \%)$ & $15(40.5 \%)$ & $38(32.5 \%)$ & \\
\hline Malnutrition & $78(50.6 \%)$ & $3(8.1 \%)$ & $75(64.1 \%)$ & \\
\hline \multirow[t]{2}{*}{ Barthel Index } & 90.0 & 5.0 & 95.0 & $<0.000^{*}$ \\
\hline & $(30.0-100.0)$ & $(0.0-15.0)$ & $(86.3-100.0)$ & \\
\hline Dysphagia severity scale & $7(5.0-7.0)$ & $3(3.0-3.0)$ & $7(7.0-7.0)$ & \\
\hline Functional oral intake scale & $7(6.0-7.0)$ & $4(2.0-5.0)$ & $7(7.0-7.0)$ & $<0.000^{*}$ \\
\hline
\end{tabular}

Results are expressed as mean \pm SD for the unpaired-t test and medium (interquartile range) for the Wilcoxon signed-rank test; Chi-square test was performed for sex, dwelling, common disease and MNA-SF.

MNA-SF, mini nutritional assessment short form.

*statistically significant. 
include at least 116 patients, with a power of 0.8 , an effect size of $d=$ 0.62 , and an alpha of 0.05 .

\section{Results}

The diseases that required long-term care included cerebral vascular disorders $(\mathrm{n}=61,39.6 \%)$, dementia $(\mathrm{n}=$ $16,10.4 \%)$, Parkinson's disease $(\mathrm{n}=8,5.2 \%)$, respiratory diseases $(\mathrm{n}=7,4.5 \%)$, cardiovascular diseases $(\mathrm{n}=4$, $2.6 \%)$, bone and joint diseases $(\mathrm{n}=28,18.2 \%)$, cancer $(\mathrm{n}=$ $4,2.6 \%)$, and others $(\mathrm{n}=27,17.5)$. The MNA-SF results showed that 78 participants $(50.6 \%)$ were malnourished and 53 participants $(34.4 \%)$ were at risk of malnutrition, while 23 participants $(14.9 \%)$ had a normal nutritional status. Based on the DSS evaluations, 37 participants had dysphagia. The median and interquartile range (IQR) of DSS scores in the dysphagia group was 3 (3.0-3.0); the median and IQR of DSS scores in the group without dysphagia was 7 (7.0-7.0). Table 1 shows that there were significant differences in sex $(p=0.053)$, diseases requiring long-term care $(\mathrm{p}<0.001)$, BMI $(\mathrm{p}<0.001)$, MNA-SF $(\mathrm{p}<0.001)$, BI $(\mathrm{p}<$ $0.001), C C(p<0.001)$, and FOIS $(p<0.001)$ findings between participants with and without dysphagia. Table 2 shows the significant differences in diseases requiring longterm care $(\mathrm{p}<0.001)$, BMI $(\mathrm{p}<0.001)$, MNA-SF $(\mathrm{p}<$ $0.001)$, BI $(\mathrm{p}<0.001), \mathrm{CC}(\mathrm{p}<0.001)$, and FOIS $(\mathrm{p}<$ $0.001)$ between men and women. Table 3 shows that CC was significantly correlated with DSS $(\mathrm{r}=0.612)$, BI $(\mathrm{r}=$ $0.565)$, MNA $(r=0.569)$, FOIS $(r=0.634)$, and BMI $(r=$ 0.800 ). The CC cut-off value for the presence of dysphagia was $31.0 \mathrm{~cm}$ in men (sensitivity, 0.818 ; specificity, 0.868 , positive predictive value, 0.563 ; negative predictive value, 0.958 ) and $29.3 \mathrm{~cm}$ in women (sensitivity, 0.760 ; specificity, 0.859 , positive predictive value, 0.667 ; negative predictive value, 0.902) (Table 4). The area under the ROC curves for $\mathrm{CC}$ was 0.876 in men and 0.842 in women (Figs. 1 and 2). Logistic regression analysis showed that the presence of dysphagia was independently associated with CC, after adjusting for age and sex (Table 5).

Table 2. Univariate analysis for sex.

\begin{tabular}{|c|c|c|c|c|}
\hline & $\begin{array}{c}\text { Total } \\
(\mathrm{n}=154)\end{array}$ & $\begin{array}{c}\text { Men } \\
(n=65)\end{array}$ & $\begin{array}{l}\text { Women } \\
(\mathrm{n}=89)\end{array}$ & $p$ value \\
\hline Age & $80.1 \pm 7.1$ & $78.8 \pm 7.1$ & $81.0 \pm 7.0$ & 0.055 \\
\hline $\begin{array}{l}\text { Disease requiring long term } \\
\text { care }\end{array}$ & & & & $<0.000^{*}$ \\
\hline \multicolumn{5}{|l|}{ n (\%) } \\
\hline Cerebral vascular disease & $61(39.6 \%)$ & $35(53.8 \%)$ & $26(29.2 \%)$ & \\
\hline Dementia & $16(10.4 \%)$ & $3(4.6 \%)$ & $13(14.6 \%)$ & \\
\hline Parkinson disease & $8(5.2 \%)$ & $4(6.2 \%)$ & $4(4.5 \%)$ & \\
\hline Respiratory disease & $7(4.5 \%)$ & $2(3.1 \%)$ & $5(5.6 \%)$ & \\
\hline Cardiovascular disease & $4(2.6 \%)$ & $3(4.6 \%)$ & $1(1.1 \%)$ & \\
\hline Bone and joint disease & $28(18.2 \%)$ & $8(12.3 \%)$ & $20(22.5 \%)$ & \\
\hline Cancer & $4(2.6 \%)$ & $3(4.6 \%)$ & $1(1.1 \%)$ & \\
\hline The Other & $27(17.5 \%)$ & $7(10.8 \%)$ & $20(22.5 \%)$ & \\
\hline \multirow[t]{2}{*}{ Body mass index } & 22.12 & 23.1 & 21.6 & $<0.000^{*}$ \\
\hline & $(19.6-25.3)$ & $(20.8-25.4)$ & $(19.2-24.3)$ & \\
\hline \multirow[t]{2}{*}{ Calf circumference $(\mathrm{cm})$} & 32.8 & 34.0 & 31.5 & $<0.000^{*}$ \\
\hline & $(29.1-35.1)$ & $(30.7-36.6)$ & $(27.7-34.1)$ & \\
\hline MNA-SF, n (\%) & & & & $<0.000^{*}$ \\
\hline Normal & $23(14.9 \%)$ & $9(13.8 \%)$ & $14(15.7 \%)$ & \\
\hline at risk & $53(34.4 \%)$ & $19(29.2 \%)$ & $34(38.2 \%)$ & \\
\hline Malnutrition & $78(50.6 \%)$ & $37(56.9 \%)$ & $41(46.1 \%)$ & \\
\hline \multirow[t]{2}{*}{ Barthel Index } & 90 & 95.0 & 90.0 & $<0.000^{*}$ \\
\hline & $(30.0-100.0)$ & $(57.5-100.0)$ & $(15.0-100.0)$ & \\
\hline Dysphagia severity scale & $7(5.0-7.0)$ & $7(6.0-7.0)$ & $7(3.0-7.0)$ & \\
\hline Functional oral intake scale & $7(6.0-7.0)$ & $7(6.0-7.0)$ & $7(5.0-7.0)$ & $<0.000^{*}$ \\
\hline
\end{tabular}

Results are expressed as mean \pm SD for the unpaired-t test and medium (interquartile range) for the Wilcoxon signed-rank test; Chi-square test was performed for sex, dwelling, common disease and MNA-SF.

MNA-SF, mini nutritional assessment short form.

*statistically significant. 
Table 3. Correlations between CC, DSS, BI, and MNA-SF.

\begin{tabular}{lccccc}
\hline & DSS & BI & MNA-SF & BMI & FOIS \\
\hline CC & $0.612^{*}$ & $0.565^{*}$ & $0.569^{*}$ & $0.800^{*}$ & $0.634^{*}$ \\
DSS & 1 & $0.810^{*}$ & $0.681^{*}$ & $0.506^{*}$ & $0.979^{*}$ \\
BI & & 1 & $0.640^{*}$ & $0.426^{*}$ & $0.808^{*}$ \\
MNA-SF & & & 1 & $0.606^{*}$ & $0.674^{*}$ \\
BMI & & & & 1 & $0.515^{*}$ \\
\hline
\end{tabular}

$* \mathrm{p}<0.05$.

CC, calf circumference; DSS, dysphagia severity scale; BI, Barthel index; MNA-SF, mini nutritional assessment short form.

Table 4. Cut-OFF value for Dysphagia in men and women.

\begin{tabular}{lcc}
\hline & \multicolumn{2}{c}{ DSS1-4 and DSS 5-7 } \\
& Men & Women \\
\hline AUC & 0.876 & 0.842 \\
Cut-OFF value & 31.000 & 29.300 \\
Sensitivity & 0.818 & 0.760 \\
Specificity & 0.868 & 0.859 \\
Positive predictive value & 0.563 & 0.667 \\
Negative predictive value & 0.958 & 0.902 \\
\hline
\end{tabular}

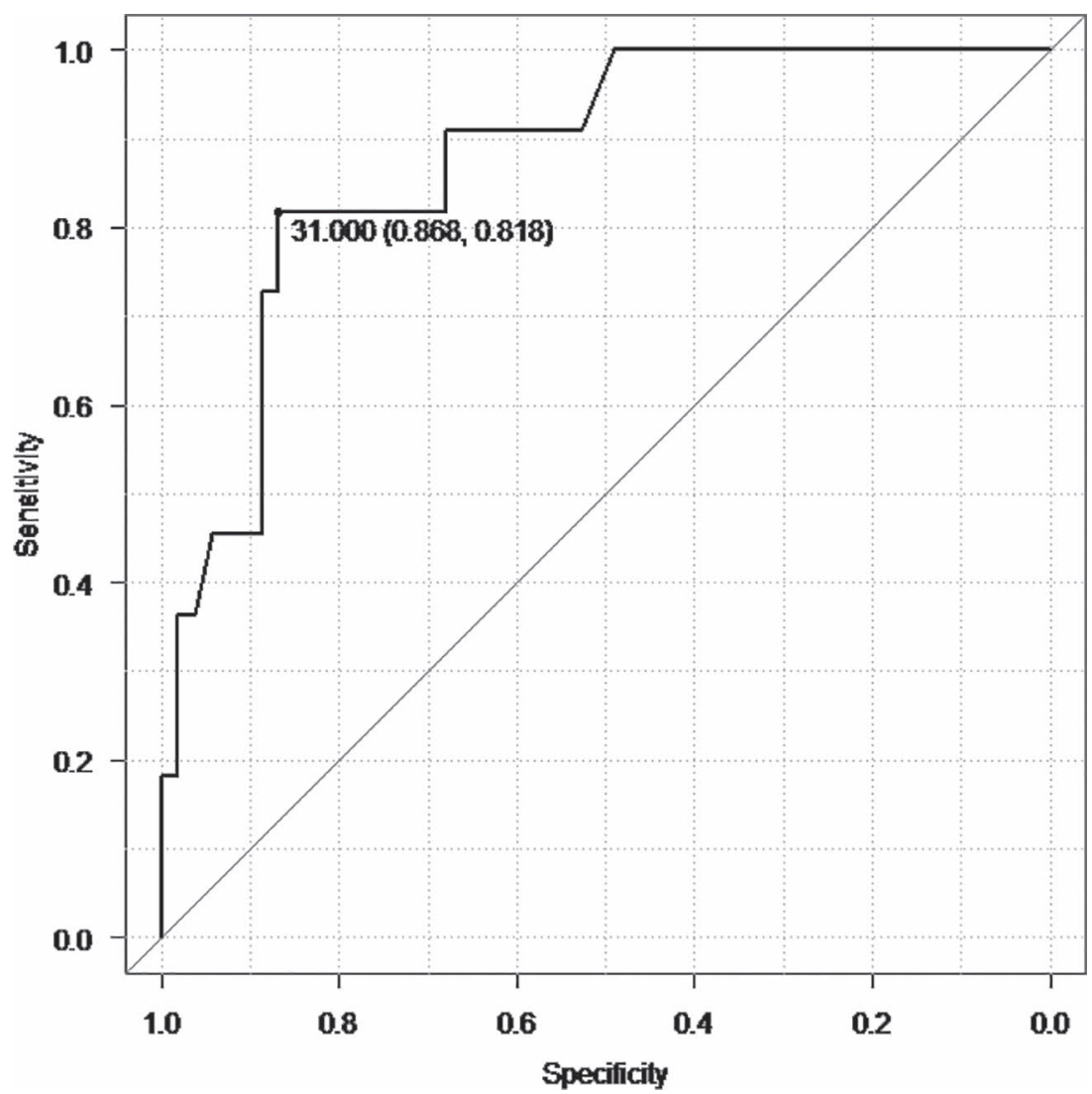

Fig. 1. Receiver operating characteristic (ROC) curve of calf circumference in men with dysphagia.

The optimal cut-off value was $31.0 \mathrm{~cm}$ (sensitivity, 0.818 ; specificity, 0.868 , positive predictive value, 0.563 ; negative predictive value, 0.958). The area under the ROC curve was 0.876 . 


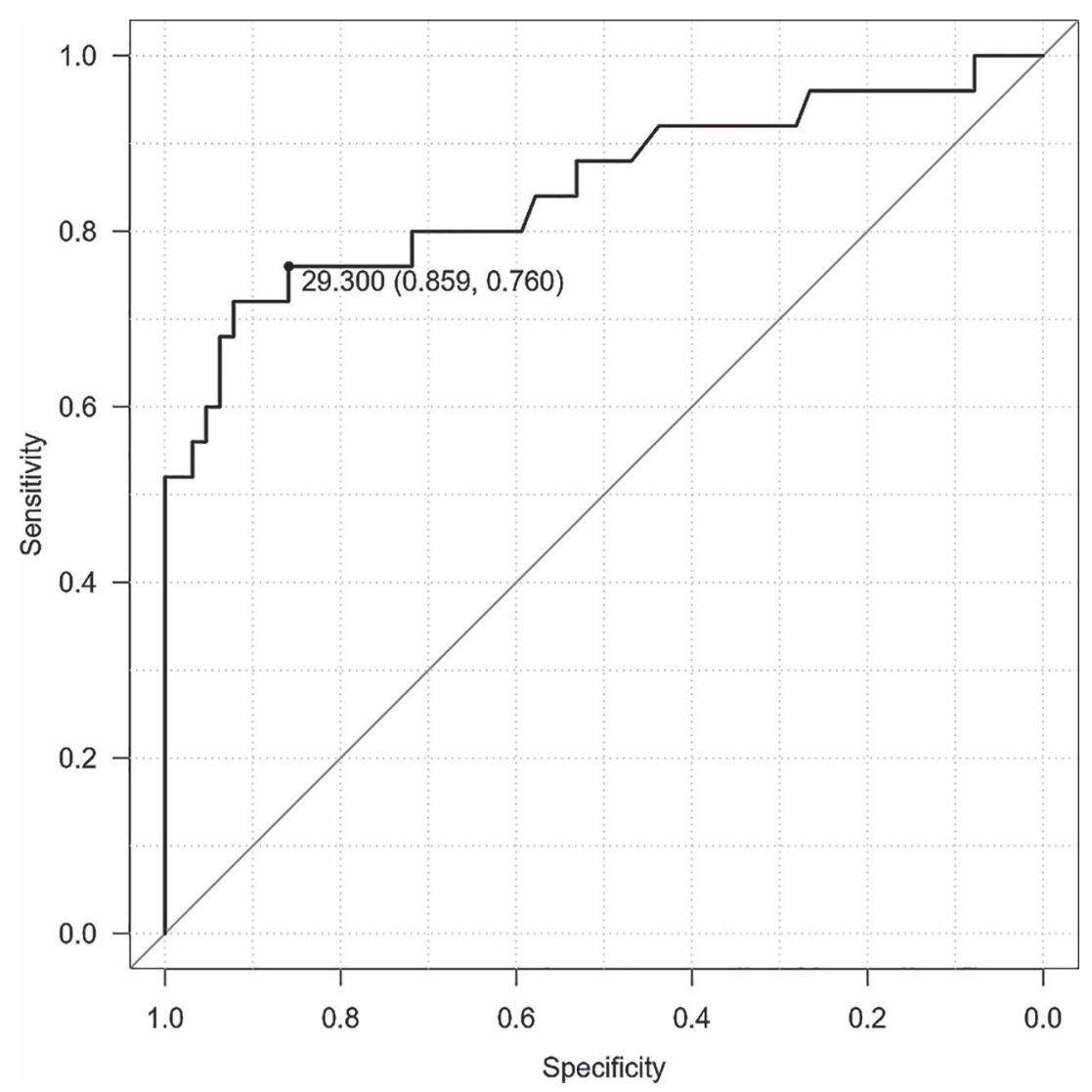

Fig. 2. Receiver operating characteristic (ROC) curve of calf circumference in women with dysphagia.

The optimal cut-off value was $29.3 \mathrm{~cm}$ (sensitivity, 0.760; specificity, 0.859, positive predictive value, 0.667 ; negative predictive value, 0.902 ). The area under the ROC curve was 0.842 .

Table 5. Logistic analysis for the presence of dysphagia.

\begin{tabular}{lcccc}
\hline & Odd ratio & \multicolumn{2}{c}{$\begin{array}{c}\text { interval } \\
\text { infidential }\end{array}$} & p value \\
\hline Cutoff value of Calf circumference & 18.315 & 6.739 & 49.781 & $0.000^{*}$ \\
Age & 1.014 & 0.948 & 1.085 & 0.677 \\
Cerebral vascular disease & 3.789 & 1.355 & 10.592 & $0.011^{*}$ \\
Parkinson disease & 3.097 & 0.302 & 31.782 & 0.341 \\
Sex & 0.451 & 0.166 & 1.233 & 0.117 \\
\hline
\end{tabular}

*statistically significant.

DSS, Dysphagia severity scale.

\section{Discussion}

The present study showed that $\mathrm{CC}$ was significantly correlated with the presence of dysphagia. In addition, $\mathrm{CC}$ had a high sensitivity and specificity for predicting dysphagia in both men and women.

\section{Relationship between CC and dysphagia}

The concept of sarcopenic dysphagia has recently been introduced (Fujishima et al. 2019). The underlying mechanism of dysphagia caused by sarcopenia is thought to be similar to secondary sarcopenia in terms of generalized skeletal muscle weakness related to inactivity, malnutrition, and other diseases. (Wakabayashi 2014; Wakabayashi and Sakuma 2014; Fujishima et al. 2019). A study regarding sarcopenic dysphagia found that the relationship between sarcopenia and dysphagia can be explained by two patterns; one in which whole-body sarcopenia is followed by a swallowing disorder, and one in which the swallowing disorder is followed by malnutrition/whole-body sarcopenia (Fujishima et al. 2019). Furthermore, based on the diagnostic criteria of sarcopenic dysphagia, patients with this disease do not have clear causative etiology related to their swallowing disorders. Since the population of the present 
study included patients with cerebral vascular diseases and Parkinson's disease, the dysphagia experienced by patients in the present study cannot be defined as sarcopenic dysphagia. However, many studies have reported dysphagia in participants with clear causative diseases associated with loss of skeletal muscle, physical dependency, and malnutrition (Hudson et al. 2000; Kuroda and Kuroda 2012; Murakami et al. 2015; Tsai and Chang 2017).

There are two possible explanations for the association between $\mathrm{CC}$ and dysphagia in the present study. First, when diseases such as cerebral vascular disease or Parkinson's disease impair swallowing mechanisms, such as through the decline of swallowing muscle strength, whole-body skeletal muscles also become paralyzed or impaired by the disease. This may result in the loss of skeletal muscles and a decline in ADLs. Second, swallowing muscles might be affected by malnutrition and/or inactivity due to low ADLs. Considering the correlation of $\mathrm{CC}$ with MNA-SF $(r=0.569)$ and $\mathrm{BI}(\mathrm{r}=0.565)$ noted in the present study, it is reasonable to infer that CC could be a useful indicator of dysphagia. In addition, a previous study that found an independent correlation between CC and dysphagia among older people in an acute hospital setting supports our results (Matsuo and Yoshimura 2018).

\section{Calf circumference is useful for dysphagia screening}

Several dysphagia screening tests have been developed to predict aspiration while swallowing liquids or semi-solids, and these tests evaluate abnormal symptoms, such as loss of swallowing after intake of a test food, coughing, and the presence of a "wet voice" after swallowing (DePippo et al. 1992; Tohara et al. 2003). However, these tests have not focused on the loss of muscle due to sarcopenia. A rapid assessment of participants with dysphagia requires a simple, time-saving screening tool that can be used before extensive clinical and instrumental examination. $\mathrm{CC}$ measurement can be performed simply, quickly, non-invasively, and inexpensively to screen for dysphagia. The present study demonstrated that the CC cut-off values for predicting dysphagia were $31.0 \mathrm{~cm}$ in men and $29.3 \mathrm{~cm}$ in women. In contrast, it has been reported that CC cut-off values for malnutrition in 1,164 Japanese hospitalized elderly people aged $>65$ years are $28.0 \mathrm{~cm}$ in men and $26.0 \mathrm{~cm}$ in women, which are smaller than the values for dysphagia found in the present study (Maeda et al. 2017). Thus, dysphagia might be followed by a worsening of nutritional status. Therefore, it is essential to screen elderly people who require long-term care for dysphagia and actively perform dysphagia rehabilitation in order to prevent advancement care requirements.

\section{Limitations}

Even though our results reveal new findings, there are some limitations. First, we excluded participants with neuromuscular diseases and bulbar palsy. Therefore, this screening test should not be applied to participants in whom the pharyngeal and laryngeal muscles might be paralyzed or impaired. Second, we did not investigate the relationship between swallowing muscle mass and $\mathrm{CC}$. Therefore, further studies that investigate these items are needed. Third, we did not perform VE in all participants, even though we performed several comprehensive dysphagia assessments. Therefore, the presence of silent aspiration was not able to be assessed in the present study. Future studies should consider a screening test for predicting silent aspiration, such as a cough test combined with CC measurement for dysphagia screening (Sato et al. 2012). In addition, the participants in the present study were recruited from a single dental hospital. Thus, sampling bias was present in the enrollment of our participants. To generalize the results of this study, further research involving a larger sample size and a multicenter study design (including a medical hospital) is required.

\section{Conclusion}

The present study showed that $\mathrm{CC}$ can be a useful screening tool to predict dysphagia in elderly people who require long-term care. Furthermore, given that $\mathrm{CC}$ is a useful screening tool for malnutrition, CC measurements might allow simultaneous malnutrition and dysphagia screening (Bonnefoy et al. 2002; Maeda et al. 2017).

\section{Acknowledgments}

This work was supported by a sponsorship funding from the Good Neighbors Company, a general incorporated association.

\section{Conflict of Interest}

The authors declare no conflict of interest.

\section{References}

Baath, C., Hall-Lord, M.L., Idvall, E., Wiberg-Hedman, K. \& Wilde Larsson, B. (2008) Interrater reliability using Modified Norton Scale, Pressure Ulcer Card, Short Form-Mini Nutritional Assessment by registered and enrolled nurses in clinical practice. J. Clin. Nurs., 17, 618-626.

Bauer, J.M., Kaiser, M.J. \& Sieber, C.C. (2008) Sarcopenia in nursing home residents. J. Am. Med. Dir. Assoc., 9, 545-551.

Bleda, M.J., Bolibar, I., Pares, R. \& Salva, A. (2002) Reliability of the mini nutritional assessment (MNA) in institutionalized elderly people. J. Nutr. Health Aging, 6, 134-137.

Bonnefoy, M., Jauffret, M., Kostka, T. \& Jusot, J.F. (2002) Usefulness of calf circumference measurement in assessing the nutritional state of hospitalized elderly people. Gerontology, 48, 162-169.

Carmont, M.R., Silbernagel, K.G., Mathy, A., Mulji, Y., Karlsson, J. \& Maffulli, N. (2013) Reliability of Achilles tendon resting angle and calf circumference measurement techniques. Foot Ankle Surg., 19, 245-249.

Crary, M.A., Mann, G.D. \& Groher, M.E. (2005) Initial psychometric assessment of a functional oral intake scale for dysphagia in stroke patients. Arch. Phys. Med. Rehabil., 86, 1516-1520.

Cruz-Jentoft, A.J., Baeyens, J.P., Bauer, J.M., Boirie, Y., Cederholm, T., Landi, F., Martin, F.C., Michel, J.P., Rolland, Y., Schneider, S.M., Topinkova, E., Vandewoude, M. \& Zamboni, M.; European Working Group on Sarcopenia in Older People (2010) 
Sarcopenia: European consensus on definition and diagnosis: report of the European Working Group on Sarcopenia in Older People. Age Ageing, 39, 412-423.

DePippo, K.L., Holas, M.A. \& Reding, M.J. (1992) Validation of the 3-oz water swallow test for aspiration following stroke. Arch. Neurol., 49, 1259-1261.

Feng, X., Todd, T., Lintzenich, C.R., Ding, J., Carr, J.J., Ge, Y., Browne, J.D., Kritchevsky, S.B. \& Butler, S.G. (2013) Agingrelated geniohyoid muscle atrophy is related to aspiration status in healthy older adults. J. Gerontol. A Biol. Sci. Med. Sci., 68, 853-860.

Fujishima, I., Fujiu-Kurachi, M., Arai, H., Hyodo, M., Kagaya, H., Maeda, K., Mori, T., Nishioka, S., Oshima, F., Ogawa, S., Ueda, K., Umezaki, T., Wakabayashi, H., Yamawaki, M. \& Yoshimura, Y. (2019) Sarcopenia and dysphagia: position paper by four professional organizations. Geriatr. Gerontol. Int., 19, 91-97.

Hudson, H.M., Daubert, C.R. \& Mills, R.H. (2000) The interdependency of protein-energy malnutrition, aging, and dysphagia. Dysphagia, 15, 31-38.

Ikeda, K., Aoyagi, Y. \& Tsubahara, A. (2007) Examination of inter-class reliability of the dysphagia severity scale. Jpn. J. Rehabil. Med., 44, S569 (in Japanese).

Jamaiyah, H. Jr., Geeta, A., Safiza, M.N., Wong, N.F., Kee, C.C., Ahmad, A.Z., Suzana, S., Rahmah, R., Khor, G.L., Ruzita, A.T., Chen, W.S., Rajaah, M. \& Faudzi, A. (2008) Reliability and technical error of calf circumference and mid-half arm span measurements for nutritional status assessment of ederly persons in Malaysia. Malays. J. Nutr., 14, 137-150.

Kawakami, R., Murakami, H., Sanada, K., Tanaka, N., Sawada, S.S., Tabata, I., Higuchi, M. \& Miyachi, M. (2015) Calf circumference as a surrogate marker of muscle mass for diagnosing sarcopenia in Japanese men and women. Geriatr. Gerontol. Int., 15, 969-976.

Kawashima, K., Motohashi, Y. \& Fujishima, I. (2004) Prevalence of dysphagia among community-dwelling elderly individuals as estimated using a questionnaire for dysphagia screening. Dysphagia, 19, 266-271.

Kayser-Jones, J. \& Pengilly, K. (1999) Dysphagia among nursing home residents. Geriatr. Nurs., 20, 77-82; quiz 84.

Kuroda, Y. \& Kuroda, R. (2012) Relationship between thinness and swallowing function in Japanese older adults: implications for sarcopenic dysphagia. J. Am. Geriatr. Soc., 60, 1785-1786.

Machida, N., Tohara, H., Hara, K., Kumakura, A., Wakasugi, Y., Nakane, A. \& Minakuchi, S. (2017) Effects of aging and sarcopenia on tongue pressure and jaw-opening force. Geriatr. Gerontol. Int., 17, 295-301.

Maeda, K. \& Akagi, J. (2016) Sarcopenia is an independent risk factor of dysphagia in hospitalized older people. Geriatr. Gerontol. Int., 16, 515-521.

Maeda, K., Koga, T., Nasu, T., Takaki, M. \& Akagi, J. (2017) Predictive accuracy of calf circumference measurements to detect decreased skeletal muscle mass and European society for clinical nutrition and metabolism-defined malnutrition in hospitalized older patients. Ann. Nutr. Metab., 71, 10-15.

Mahoney, F.I. \& Barthel, D.W. (1965) Functional evaluation: the Barthel Index. Md. State Med. J., 14, 61-65.

Matsuo, H. \& Yoshimura, Y. (2018) Calf circumference is associated with dysphagia in acute-care inpatients. Geriatr. Nurs., 39, 186-190.

Matsuo, H., Yoshimura, Y., Ishizaki, N. \& Ueno, T. (2017) Dysphagia is associated with functional decline during acutecare hospitalization of older patients. Geriatr. Gerontol. Int.,
17, 1610-1616.

Molfenter, S.M., Amin, M.R., Branski, R.C., Brumm, J.D., Hagiwara, M., Roof, S.A. \& Lazarus, C.L. (2015) Age-related changes in pharyngeal lumen size: a retrospective MRI analysis. Dysphagia, 30, 321-327.

Murakami, K., Hirano, H., Watanabe, Y., Edahiro, A., Ohara, Y., Yoshida, H., Kim, H., Takagi, D. \& Hironaka, S. (2015) Relationship between swallowing function and the skeletal muscle mass of older adults requiring long-term care. Geriatr. Gerontol. Int., 15, 1185-1192.

Nishimura, K., Kagaya, H., Shibata, S., Onogi, K., Inamoto, Y., Ota, K., Miki, T., Tamura, S. \& Saioh, E. (2015) Accuracy of dysphagia severity scale rating without using videoendoscopic evaluation of swallowing. Jpn. J. Compr. Rehabil. Sci., 6, 124-128.

Perissinotto, E., Pisent, C., Sergi, G. \& Grigoletto, F.; ILSA Working Group (2002) Anthropometric measurements in the elderly: age and gender differences. Br. J. Nutr., 87, 177-186.

Portero-McLellan, K.C., Staudt, C., Silva, F.R., Delbue Bernardi, J.L., Baston Frenhani, P. \& Leandro Mehri, V.A. (2010) The use of calf circumference measurement as an anthropometric tool to monitor nutritional status in elderly inpatients. J. Nutr. Health Aging, 14, 266-270.

Rubenstein, L.Z., Harker, J.O., Salva, A., Guigoz, Y. \& Vellas, B. (2001) Screening for undernutrition in geriatric practice: developing the short-form mini-nutritional assessment (MNASF). J. Gerontol. A Biol. Sci. Med. Sci., 56, M366-372.

Sainsbury, A., Seebass, G., Bansal, A. \& Young, J.B. (2005) Reliability of the Barthel Index when used with older people. Age Ageing, 34, 228-232.

Sato, M., Tohara, H., Iida, T., Wada, S., Inoue, M. \& Ueda, K. (2012) Simplified cough test for screening silent aspiration. Arch. Phys. Med. Rehabil., 93, 1982-1986.

Tamura, F., Kikutani, T., Tohara, T., Yoshida, M. \& Yaegaki, K. (2012) Tongue thickness relates to nutritional status in the elderly. Dysphagia, 27, 556-561.

Tohara, H., Saitoh, E., Mays, K.A., Kuhlemeier, K. \& Palmer, J.B. (2003) Three tests for predicting aspiration without videofluorography. Dysphagia, 18, 126-134.

Tsai, H.J. \& Chang, F.K. (2017) Associations between body mass index, mid-arm circumference, calf circumference, and functional ability over time in an elderly Taiwanese population. PLoS One, 12, e 0175062 .

United Nations (2015) World population prospects: 2015 revision. http://www.un.org/en/development/desa/publications/worldpopulation-prospects-2015-revision.html [Accessed: August 29, 2018].

Vellas, B., Villars, H., Abellan, G., Soto, M.E., Rolland, Y., Guigoz, Y., Morley, J.E., Chumlea, W., Salva, A., Rubenstein, L.Z. \& Garry, P. (2006) Overview of the MNA: its history and challenges. J. Nutr. Health Aging, 10, 456-463; discussion 463-455.

Wakabayashi, H. (2014) Presbyphagia and sarcopenic dysphagia: association between aging, sarcopenia, and deglutition disorders. J. Frailty Aging, 3, 97-103.

Wakabayashi, H. \& Matsushima, M. (2016) Neck circumference is not associated with dysphagia but with undernutrition in elderly individuals requiring long-term care. J. Nutr. Health Aging, 20, 355-360.

Wakabayashi, H. \& Sakuma, K. (2014) Rehabilitation nutrition for sarcopenia with disability: a combination of both rehabilitation and nutrition care management. J. Cachexia Sarcopenia Muscle, 5, 269-277. 